Cita bibliográfica: Garulo Ramón, C., Díez Sánchez, J. J. y Sellers Rubio, R. (2021). La influencia de los factores políticos en la externalización de servicios de información turística. Investigaciones Turísticas (22), pp. 254-276. https://doi.org/10.14198/INTURI2021.22.11

\title{
La influencia de los factores políticos en la externalización de servicios de información turística
}

\author{
The influence of political factors in the outsourcing of tourist information services
}

Carlos Garulo Ramón iD, Universidad de Alicante, España. IMEP, centro adscrito UMH, España. cgarulor@gmail.com

Juan José Díez Sánchez iD, Universidad de Alicante, España

juanjo.diez@ua.es

Ricardo Sellers Rubio iD, Universidad de Alicante, España

ricardo.sellers@ua.es

\section{RESUMEN}

La legislación española establece, como norma general, que las administraciones públicas deberán prestar los servicios públicos con sus propios medios y que, solo cuando carezcan de éstos, podrán externalizar la prestación de determinados servicios. En este sentido, el servicio de información turística es uno de los servicios que las administraciones tienden a externalizar en mayor medida al requerir de una mayor flexibilidad horaria para atender a las necesidades de los turistas. Así, el objetivo general de este trabajo se centra en analizar la potencial influencia que pudieran tener determinados factores políticos en la propensión a externalizar los servicios de información turística a nivel municipal. A tal efecto, se ha diseñado una investigación que recoge la situación de los servicios de información turística en la provincia de Alicante durante el año 2019. Los resultados obtenidos indican que no existe una mayor o menor propensión a externalizar en función del partido político que gobierna ni en base al partido que tiene las competencias en materia de turismo dentro de la corporación municipal. Sin embargo, los resultados señalan que la forma de gobernanza sí ejerce una influencia, de forma que las corporaciones en las que se gobierna con una mayoría absoluta tienden a externalizar los servicios de información en mayor medida que las corporaciones con un gobierno de coalición. Estos resultados sugieren que los factores de externalización podrían no corresponderse con las potenciales reducciones de coste o mejoras en la eficiencia que aducen algunas corporaciones para externalizar dichos servicios. 
Palabras clave: Externalización; servicios; contratación pública; factores políticos; información turística.

\section{ABSTRACT}

As a general rule, Spanish legislation establishes that public administrations must provide public services with their own means and that, only when they lack these, may they outsource the provision of certain services. In this sense, the tourist information service is one of the services that administrations tend to outsource to a greater extent as they require greater time flexibility to meet the needs of tourists. Thus, the overall objective of this study is to analyse the potential influence that certain political factors could have on the propensity to outsource tourist information services at the municipal level. As such, an investigation has been designed that researches the state of tourist information services in the province of Alicante during 2019. The results obtained indicate that there is no greater or lesser propensity to outsource depending on the political party that governs or on the basis of the party that has powers in tourism within the municipal corporation. However, the results indicate that the form of governance does exert an influence, whereby corporations governed by an absolute majority tend to outsource information services to a greater extent than corporations with a coalition government. These results suggest that outsourcing factors might not correspond to the potential cost reductions or improvements in efficiency that some corporations claim as justification for outsourcing such services.

Keywords: Outsourcing; services; public procurement; political factors; tourist information.

\section{INTRODUCCIÓN}

España se ha situado, durante 2018, en el segundo puesto en cuanto a llegadas de turistas internacionales (solo superado por Francia) y también en cuanto a ingresos por turismo internacional, donde solo ha sido superada por Estados Unidos (OMT-UNWTO, 2018). Así, el sector turístico ha supuesto durante 2018 el 12,30\% del Producto Interior Bruto de España y ha generado 2,62 millones de puestos de trabajo (el 12,70\% total del empleo), mostrando una clara tendencia al alza (INE, 2018) en los últimos años y que solo se ha visto interrumpida por la crisis generada por la Covid-19 desde marzo de 2020. En cualquier caso, y dada la importancia que tiene el turismo como motor económico y de empleo, los servicios turísticos deben adaptarse, cada vez más, a las exigencias y necesidades de los turistas que visitan nuestro territorio. Ello es debido a la creciente necesidad de mantener la competitividad y el liderazgo del turismo español en el ámbito internacional.

En la actualidad, muchos de los servicios dirigidos a captar y/o mejorar la calidad de la experiencia turística son competencia de las diferentes administraciones públicas. En este sentido, la legislación vigente (Ley 9/2017, de Contratos del Sector Público) establece como regla general que la prestación de servicios será realizada por la propia Administración con sus propios medios y que, cuando carezca de éstos, podrá externalizar determinados servicios. En este sentido, determinadas administraciones tienden a que determinados servicios sean prestados directamente por un tercero al tener dificultades para su gestión directa, aludiendo 
motivos organizativos, falta de personal o flexibilidad horaria, entre otros aspectos (tal y como se desprende de los diferentes informes de insuficiencia de medios publicados en los expedientes de licitación conforme a lo establecido en el art.63.3.a. de la LCSP) ${ }^{1}$.

Dentro de los servicios turísticos prestados por las administraciones merece especial relevancia el servicio de información turística. Este servicio se presta habitualmente a través de las oficinas y puntos de información turística, las cuales se constituyen en un servicio esencial en los destinos turísticos al permitir a las autoridades locales, brindar a los turistas una acogida en nombre de las empresas y los ciudadanos del destino (Mirabell, 2011). Por tanto, este servicio se constituye en uno de los principales canales para garantizar que los turistas disponen del conocimiento de la oferta turística de un destino o territorio, siendo el nivel municipal la principal herramienta o punto de contacto para informar a los turistas de toda la oferta del destino y considerando a la administración local como la pieza clave en la prestación de servicios a los ciudadanos ya que los municipios son los escalones más cercanos que permiten canalizar la participación del ciudadano en los temas públicos (Pina y Torres, 2002)

En el caso concreto de la Comunidad Valenciana se ha desarrollado una estructura o "red" propia, denominada Red Tourist Info, a la que se adhieren los municipios y que sirve para garantizar una imagen única del territorio bajo unos estándares de información y calidad propios. La coordinación de todas ellas es importante para garantizar que los turistas puedan tener acceso a la información que puedan necesitar tanto del destino local, como del resto de destinos de la Comunidad Valenciana como destino regional.

Precisamente, el servicio de información turística es uno de los servicios turísticos que las administraciones públics tienden a externalizar, aumentándose el número de licitaciones desde el año 2018 (Garulo et. al., 2020). Aunque la externalización de servicios tiene asociadas ciertas ventajas e inconvenientes relacionados con el coste de los mismos (Bel, 2009; Martínez Rodríguez, 2003; Salvador y Riba, 2017; Valencia, 2019), la flexibilidad (Ramió y García Codina, 2006) o presiones fiscales (Bel, 2009; Martínez Rodríguez, 2003; Salvador y Riba, 2017), algunos autores como Bel (2009), Martínez Rodríguez (2003) o Valencia (2019) también defienden, entre otras causas, el factor político como uno de los principales argumentos para la externalización o no de los servicios prestados por parte de las administraciones públicas.

En este sentido, y como novedad en la literatura académica, el objetivo principal de este trabajo se centra en analizar la potencial influencia que pudieran tener determinados factores políticos como variables explicativas de la externalización de los servicios de información turística desde una triple vertiente: en primer lugar, en función del partido político que ostenta la alcaldía; en segundo lugar, en función del partido político que sustenta la competencia en turismo municipal; $y$, en tercer lugar, en función de si la corporación municipal gobierna en

\footnotetext{
1. Algunos informes de insuficiencia de medios consultados:

182/2020 Servicio de Atención, Información y Colaboración en las tareas de Promoción Turística del Municipio de Tijarafe (Cláusula 4.a): https://n9.cl/3trtf

74/2020 Servicio de información turística de la ciudad de Málaga: https://n9.cl/iiu7 CONT-12/2019 Servicio de gestión de la Oficina de Turismo de Sueca: $\underline{\mathrm{https}: / / \mathrm{n} 9 . \mathrm{cl} / \mathrm{d} 9 \mathrm{w} 0 \mathrm{o}}$

27/2019 Servicio de información turística y gestión de la oficina de información turística de Talavera de la Reina: https://n9.cl/i7xn
} 
mayoría o en coalición o alianza. Además, se pretende conocer la opinión de los municipios respecto a diferentes aspectos sobre la externalización del servicio de información turística y la gestión interna por parte de los ayuntamientos. A tal efecto, se ha diseñado una investigación que recoge la situación de los servicios de información turística en la provincia de Alicante.

\section{MARCO TEÓRICO}

\subsection{Competencias turísticas por parte de las administraciones públicas}

La Constitución Española de 1978 establece las competencias generales del Estado (art. 149) y de las Comunidades Autónomas (art. 148). "El modelo de Estado compuesto definido en la Constitución de 1978 deja en manos de los Entes territoriales: Comunidades Autónomas y Entes Locales, la ordenación y promoción del turismo en el territorio correspondiente" (Rodríguez-Arana, 2008, p.370). Dentro de los principios rectores de la política social y económica, y siguiendo a Fernández Rodríguez (2016), llama la atención de que no exista una referencia al turismo en ninguno de sus preceptos, aunque muy probablemente los constituyentes considerasen que esta materia estaba comprendida dentro del ocio. Este hecho, unido a la transversalidad del turismo, ha generado múltiples problemas de distribución competencial entre Estado y Comunidades Autónomas. Un ejemplo de ello es la STC 125/1984, de 20 de diciembre, donde según el art.148.1.18 de la C.E., las Comunidades Autónomas podrán asumir competencias sobre promoción y ordenación del turismo en su ámbito territorial, sin que el art.149 de la C.E. contenga ninguna reserva competencial en favor del Estado sobre turismo. Además, conviene destacar que, en ocasiones, los conceptos recogidos en las normas constitucionales y estatutarias relativas a la distribución de competencias clasifican la realidad social en materias para ordenar esa distribución, pero en ocasiones poseen un inevitable grado de indeterminación que puede dar lugar a que una misma materia tenga dimensiones que puedan ser clasificables dentro de otros conceptos materiales y asumibles en otros títulos competenciales.

Por tanto, la Constitución Española de 1978 no establece en sí una competencia exclusiva en materia de turismo, pero sí establece otras que de cierta manera influye en la actividad turística como por ejemplo, en su art.40 donde establece que "Ios poderes públicos promoverán las condiciones favorables para el progreso social y económico..." (Constitución Española, 1978); o en su art.149, donde a pesar de que no establece como tal una competencia específica de turismo, sí que menciona una serie de competencias que indirectamente inciden sobre la actividad turística, y todas ellas relacionadas con infraestructuras de transporte, patrimonio histórico-artístico, comunicaciones, medio ambiente o inmigración (entre otras).

Así pues, el turismo se ha convertido, de acuerdo con Villanueva Cuevas (2016, p.170) "en una de las materias que las Comunidades Autónomas pudieron asumir en sus Estatutos de Autonomía desde el mismo momento de aprobación de éstos". Esto hizo que las Comunidades Autónomas hayan adquirido prácticamente en exclusiva, la competencia de turismo y el Estado solamente posea algunas competencias de manera residual. Por tanto, en base a los diferentes tipos de competencias definidas por Guerra y Martín (2009), la competencia en materia de turismo podemos calificarla como competencia «exclusiva absoluta», es decir, 
que la titularidad de la totalidad de las funciones y potestades públicas en relación con dicha materia recae en exclusiva, sobre las Comunidades Autónomas.

Tabla 1: Competencias Autonómicas relacionadas con la actividad turística

\begin{tabular}{|c|c|}
\hline Constitución Española 1978 (art.149) & $\begin{array}{c}\text { Ley Orgánica 1/2006 de Estatuto de la } \\
\text { Comunidad Valenciana }\end{array}$ \\
\hline $\begin{array}{l}\text { Promoción y ordenación del turismo en su } \\
\text { ámbito territorial. }\end{array}$ & Turismo \\
\hline Museos de interés para la C.Autónoma. & $\begin{array}{l}\text { Museos que no sean de titularidad estatal. } \\
\text { Centros dramáticos y servicios de Bellas Artes de } \\
\text { interés para la Comunitat Valenciana. }\end{array}$ \\
\hline $\begin{array}{l}\text { Patrimonio monumental de interés de la } \\
\text { C.Autónoma. }\end{array}$ & $\begin{array}{l}\text { Patrimonio histórico, artístico, monumental, } \\
\text { arquitectónico, arqueológico y científico, sin } \\
\text { perjuicio de lo establecido en la Constitución } \\
\text { Española. }\end{array}$ \\
\hline \multirow[t]{2}{*}{ Fomento de la cultura. } & Cultura. \\
\hline & Espectáculos. \\
\hline $\begin{array}{c}\text { Promoción del deporte y de la adecuada } \\
\text { utilización del ocio. }\end{array}$ & Deporte y ocio. \\
\hline La artesanía. & La Artesanía. \\
\hline $\begin{array}{l}\text { Organización de sus instituciones de } \\
\text { autogobierno }\end{array}$ & $\begin{array}{l}\text { Organización de sus instituciones de } \\
\text { autogobierno }\end{array}$ \\
\hline $\begin{array}{c}\text { Obras públicas de interés para la C.Autónoma en } \\
\text { su propio entorno. }\end{array}$ & $\begin{array}{c}\text { Obras públicas que no tengan la calificación de } \\
\text { interés general del Estado o cuya relación no } \\
\text { afecte a otra C.Autónoma. }\end{array}$ \\
\hline $\begin{array}{l}\text { Ferrocarriles y carreteras cuyo itinerario se } \\
\text { desarrolle íntegramente en el territorio de } \\
\text { la C.Autónoma y en los mismos términos, el } \\
\text { transporte desarrollado por estos medios o por } \\
\text { cable. Puertos de refugio, puertos y aeropuertos } \\
\text { deportivos, y en general, los que no desarrollen } \\
\text { actividades comerciales. }\end{array}$ & $\begin{array}{l}\text { Carreteras y caminos cuyo itinerario transcurra } \\
\text { íntegramente dentro del territorio de la } \\
\text { Comunitat Valenciana. Ferrocarriles, transportes } \\
\text { terrestres, marítimos, fluviales y por cable; } \\
\text { puertos, aeropuertos, helipuertos, sin perjuicio } \\
\text { de lo establecido en la Constitución Española. }\end{array}$ \\
\hline Los montes y aprovechamientos forestales & $\begin{array}{c}\text { Montes, aprovechamientos y servicios } \\
\text { forestales, vías pecuarias y pastos, espacios } \\
\text { naturales protegidos y tratamiento especial de } \\
\text { zonas de montaña, de acuerdo a lo establecido } \\
\text { en la Constitución Española. }\end{array}$ \\
\hline Ordenación del territorio, urbanismo y vivienda. & $\begin{array}{l}\text { Ordenación del territorio y del litoral, urbanismo } \\
\text { y vivienda. }\end{array}$ \\
\hline \multicolumn{2}{|l|}{$\begin{array}{l}\text { La gestión en materia de protección del medio } \\
\text { ambiente. }\end{array}$} \\
\hline Ferias interiores & \\
\hline
\end{tabular}

Elaboración propia. 
Una vez analizada la competencia turística a nivel estatal y autonómico, conviene destacar qué competencia turística corresponde a los municipios. El texto consolidado de La Ley 7/1985, reguladora de las bases de régimen local, establece que los Municipios, la Provincia y las Islas, son entidades básicas de la organización territorial del Estado (art.1), estableciendo a las mismas como entidades locales (art.3). En este último artículo, también se indica que gozarán de la condición de Entidad Local, las Comarcas u otras entidades que agrupen varios Municipios, las Áreas Metropolitanas y las Mancomunidades de Municipios. Esta misma Ley, también establece que para a efectividad de la autonomía de las entidades locales, la legislación estatal y autonómica deberá asegurar el derecho de estas entidades a intervenir en cuantos asuntos afecten directamente al círculo de sus intereses, atribuyéndoles las competencias que procedan en atención a las características de la actividad pública y a la capacidad de gestión de la Entidad Local (art.2), todo ello de conformidad con los principios de descentralización, proximidad, eficacia y eficiencia, así como estabilidad presupuestaria y sostenibilidad financiera. Las entidades Locales tendrán, por tanto "plena capacidad jurídica para (...) celebrar contratos, establecer y explotar obras o servicios públicos" (art. 5), estableciendo así mismo que "son servicios públicos locales los que prestan las entidades locales en el ámbito de sus competencias" (art. 85), enumerando además dos formas de gestión de dichos servicios públicos: la gestión directa y la gestión indirecta. Como competencias puramente turísticas, dentro de esta ley se establecen las competencias de información y promoción de la actividad turística de interés y ámbito local (art.25) y la de promoción y gestión turística por delegación de las Comunidades Autónomas (art.27), a las que la Ley 8/2010, de régimen local de la Comunitat Valenciana, añade las actividades o instalaciones culturales y deportivas, la ocupación del tiempo libre y el turismo (art.33).

La administración local, por tanto, "se está configurando en los países más desarrollados, como una pieza clave en la prestación de servicios a los ciudadanos, considerando a los municipios como los escalones más cercanos que canalizan la participación del ciudadano en los temas públicos" (Pina y Torres, 2002, p.226).

Por último, cabe mencionar la competencia turística a nivel europeo. La Unión Europea también apuesta por las entidades locales y regionales para el logro de los objetivos de desarrollo del potencial turístico destacando la contribución de las autoridades locales y regionales al desarrollo turístico de la UE (Dictamen de la Comisión de las Regiones, de 13 de octubre de 1999), destacando además que el producto turístico está constituido por una serie o cadena de servicios e infraestructuras indisociables que engloban elementos como los medios de desplazamientos, estado de carreteras, transportes públicos y privados, evacuación de aguas y alcantarillado, entorno natural y urbano, infraestructura turística, calidad de vida, accesibilidad, gestión de sitios y monumentos, organización de acontecimientos culturales y recreativos, la estructuración de la información turística, y la adecuada promoción de las zonas turísticas, tanto dentro del país como en el extranjero (punto 2.2), destacando en dicho dictamen, además que "a mayor grado de competencias turísticas por parte de autoridades locales y regionales (...), mayor será el desarrollo del mismo" (punto 3.1.4.) 


\subsection{La externalización de servicios turísticos y los principales motivos para su externalización}

Para comprender mejor la externalización de servicios turísticos y los principales motivos para su externalización, debemos hacer un breve recorrido por el concepto de externalización, así como por los principales motivos analizados por diversos autores que llevan a las administraciones públicas a recurrir a esta modalidad para la prestación de determinados servicios (incluyendo los turísticos).

En primer lugar, cabe destacar que el concepto de externalización proviene de la traducción del término inglés outsourcing (Ramió y Salvador, 2012). Cuando el sector público asume la provisión pública de un servicio, debe determinar qué método de provisión será el más adecuado a sus objetivos: la producción pública o la contratación externa (Martínez Rodríguez, 2003). El primer método utiliza personal y capital público para la prestación del servicio público. La segunda de ellas consiste en contratar a un tercero (generalmente una empresa privada) aunque la responsabilidad de la prestación del servicio sigue siendo de la administración pública.

Para poder externalizar un servicio, la administración pública debe regirse por la Ley 9/2017, de 8 de noviembre, de Contratos del Sector Público, la cual establece como límite que "no podrán ser objeto de estos contratos los servicios que impliquen el ejercicio de la autoridad inherente a los poderes públicos" (art. 17). Así mismo, esta Ley, establece dos modalidades para la externalización del servicio: el contrato de servicios (art.17) y contrato de concesión de servicios (art.15), diferenciándose uno de otro en base al riesgo operacional. Si el riesgo recae en la administración, será un contrato de servicios, y si el mismo recae en la empresa adjudicataria, será un contrato de concesión de servicios.

No obstante, Bel (2009) nos indica que no debemos confundir el término externalizar con privatizar ya que en ocasiones el término privatización se utiliza para hacer referencia a la contratación externa de servicios, hecho que es cuestionado desde algunas vertientes académicas, como es el caso de la Administración pública en Europa continental, al apreciarse que en el caso de la contratación externa no se está ante una privatización en sentido estricto puesto que el sector público se reserva un importante espacio de decisiones y el contrato o concesión tiene carácter temporal y no indefinido.

Así pues, la externalización ha sido analizada por diversos autores, y pueden suponer una serie de ventajas e inconvenientes que conviene tener en cuenta. Pina y Torres (2002) indica que el control cada vez más exhaustivo del endeudamiento y del déficit público han impulsado, entre otras medidas, la privatización de empresas públicas, la descentralización de servicios y la transferencia de la gestión de servicios públicos al sector privado como forma de contener el gasto. Laguna de Paz, JC (2011), añade que la externalización es una técnica de gestión que permite a la Administración concentrar sus recursos humanos y materiales a la hora de ejercer sus competencias sin tener que asumir la ejecución y realización de determinadas actividades instrumentales. Por tanto, extrapolando lo establecido por estos autores, la externalización de servicios turísticos al sector privado puede deberse a una manera de contener el gasto, o como forma de prestar el servicio sin asumir la ejecución directa del mismo 
Otros autores como Ramió y García Codina (2006), establecen que la privatización, si se realiza bajo la premisa de que el contrato se celebra en condiciones de competencias, preexistente o inducida, permitirá una mejora de la eficacia (calidad) y eficiencia (coste), dado que la contratación de la producción del servicio se realiza a aquella organización que más competitiva sea en cuanto a costes y calidad puesto que "tiene lugar en las áreas y capacidades en que cada organización goza de ventaja competitiva al mismo tiempo que los costes internalizados, fijos, se tornan variables", con lo que tiene lugar a priori, una reducción de costes motivado principalmente a que la contratación externa reduciría (o no aumentaría), el número de empleados públicos. Estos mismos autores añaden que la contratación externa implica una "mayor flexibilidad respecto a la demanda del servicio". Estos argumentos cobran especial relevancia en el servicio de información turística, ya que permite esa flexibilidad horaria que adaptada al volumen de demanda y a las necesidades de horarios de los turistas, muy difícil de conseguir con personal funcionario.

A propósito de esto, Valencia (2019) establece que en España podemos diferenciar claramente dos modelos de externalización: un modelo reactivo, y un modelo proactivo. El primero de ellos es "disfuncional, en donde no se externaliza para mejorar la gestión sino para cubrir los déficits, y donde la administración hace un uso pasivo de sus prerrogativas de gestión". El segundo, es un modelo proactivo "de carácter normativo que busca la superación de las deficiencias del modelo anterior (...), regido por los conceptos de eficiencia y eficacia". En el caso de los servicios de información turística, y tal y como se ha señalado anteriormente, estaríamos en el primero de los escenarios ya que gran parte de las administraciones argumentan para justificar la necesidad de la contratación o externalización, que no disponen de medios suficientes para prestar el servicio.

En cuanto a las razones para la externalización de servicios por parte de las administraciones públicas, conviene añadir que la Ley $9 / 2017$, de Contratos del Sector Público, no especifica los motivos para la externalización de servicios, pero sí que establece, dentro de sus objetivos conseguir una mejor relación calidad-precio, velando por la inclusión de aspectos cualitativos, medioambientales, sociales e innovadores vinculados al objeto del contrato (preámbulo II, párrafos 1 y 2). También indica que las entidades del sector público no podrán celebrar otros contratos que aquellos que sean necesarios para el cumplimiento y realización de sus fines institucionales. A tal efecto, la naturaleza y extensión de las necesidades que pretenden cubrirse mediante el contrato proyectado, así como la idoneidad de su objeto y contenido para satisfacerlas, (...) deben ser determinadas con precisión, dejando constancia de ello en la documentación preparatoria, antes de iniciar el procedimiento encaminado a su ejecución (Art.28.1)

Así mismo, cabe destacar que esta misma normativa, establece que "la prestación de servicios se realizará normalmente por la propia Administración por sus propios medios. No obstante, cuando carezca de medios suficientes, previa la debida justificación en el expediente, se podrá contratar de conformidad con lo establecido (...) en la presente ley" (Art.30.3), siempre publicando en el perfil del contratante, la memoria justificativa del contrato y el informe de insuficiencia de medios (Art.63.3.a). 
No obstante, diversos autores afirman la existencia de otra serie de razones o motivos que justifican la externalización de los servicios por parte de la administración pública. Autores como Martínez Rodríguez (2003), Bel (2009) o Valencia (2019), defienden, dentro de los fundamentos o bases teóricas de la externalización como estrategia de gestión de servicios públicos, los factores políticos como uno de los principales motivos o razones para la externalización de los servicios por parte de las administraciones públicas.

Bel (2009) justifica este motivo atendiendo al interés utilitarista del político (ganar las elecciones), motivado e influenciado además por posibles grupos de presión con intereses particulares (principalmente sindicatos y patronales) o por la aplicación de unas políticas sobre otras en base al partido político (las formaciones de izquierda, tradicionalmente, suelen externalizar menos que las de derechas).

Sobre este concepto Martínez Rodríguez (2003) añade, además, el concepto de clientelismo político o political patronage al motivo anteriormente citado por Bel (intereses políticos y motivaciones ideológicas), añadiendo que los partidos políticos, en la medida que exista un sentimiento a favor de la intervención pública por parte de la población, pueden obtener menos votos al llevar a cabo la externalización de servicios, a pesar del posible efecto económico positivo que pueda suponer sobre el presupuesto municipal.

Por último, Valencia (2019), añade que las razones para externalizar atienden a las alianzas de poder entre élites burocráticas y políticas o a restricciones legislativas, tecnológicas o presupuestarias sin que se corresponden en muchos casos a la mejora de la eficacia y la eficiencia.

Son, por tanto, estos intereses políticos los que se configuran como el eje central de la presente investigación. No obstante, para comprender mejor los resultados obtenidos, cabría añadir que existe una tendencia generalizada a pensar que los partidos políticos de derechas tienden más a la privatización y externalización ya que suelen posicionarse más en favorecer a los empresarios al centrarse más en el individuo y la iniciativa privada. Por el contrario, se suele pensar que los partidos de izquierdas suelen centrarse más en la internalización de la gestión puesto que suelen gestionar pensando en el conjunto de la sociedad, como conjunto de personas que forman una comunidad (Junior Report, 7 de noviembre de 2019). Pero no solo la ideología política puede influir en la externalización de los servicios. También deberemos tener en cuenta la fragmentación o no de los diferentes gobiernos a la hora de externalizar un servicio y si se gobierna en coalición o en mayoría, puesto que a priori, puede influir en la decisión final de externalizar un servicio o no, así como qué partido político sustenta la competencia en materia de turismo dentro de la corporación municipal.

El objetivo principal de esta investigación, por tanto, será contrastar si en el caso de los servicios de información turística, los factores políticos influyen en la decisión o no de su externalización. Para ello, nos centraremos en los municipios y oficinas de información turística de la provincia de Alicante adheridos a la Red Tourist Info de Generalitat Valenciana. Primero, analizaremos la opinión de los municipios respecto a diferentes aspectos sobre la externalización de las oficinas y puntos de información turística y la gestión interna por parte de los Ayuntamientos, para determinar si los factores políticos se sitúan o no como uno de los 
principales argumentos o causas para la externalización del servicio. Posteriormente, detallaremos de qué manera influyen los factores políticos a la hora de externalizar el servicio analizando la propensión a externalizar el servicio de información turística en función de los 3 aspectos mencionados anteriormente: partido político que gobierna, partido político que tiene la competencia en materia de turismo dentro de la corporación municipal y, por último, si la corporación municipal gobierna en mayoría absoluta o en coalición.

\section{METODOLOGÍA}

\subsection{El Servicio de información turística}

Los sistemas de información turística son aquellos dirigidos a "satisfacer las necesidades de información, orientación y asesoramiento de sus usuarios y a promocionar el patrimonio turístico de la zona que tengan encomendada" (Morales, 2018, p.2). Estos sistemas dependen de varios parámetros como la legislación (estatal, autonómica o local) o la finalidad con la que han sido creados, pudiendo incluso comercializar productos y servicios turísticos. Siguiendo a esta misma autora, los sistemas pueden ser de titularidad pública, privada u optar por un modelo mixto, aunque cada vez es más frecuente encontrar servicios de información turística gestionados únicamente por entidades privadas.

De acuerdo con Ibañez Jarque (2001), cuando los turistas eligen un destino, definen también sus expectativas iniciales, o lo que es lo mismo, la existencia de servicios, recursos y oferta que dé respuesta al tipo de estancia que pretende. El turista, por tanto, deberá conocer cuáles son los atractivos y servicios del territorio (cómo son, dónde están y cómo se llega hasta ellos) para que se pueda establecer una correspondencia adecuada entre éstas y la realidad de su viaje.

Por tanto, a pesar de que "las oficinas de información turística no ofrecen el servicio turístico propiamente dicho, lo complementan en el marco de lo que constituye la prestación turística informativa necesaria para la prestación de un servicio turístico al consumidor que sea de suficiente calidad" (Fernández Rodríguez, 2016, p.95), constituyéndose en uno de los principales canales para garantizar la atención de peticiones de información por parte de los turistas y proporcionar el conocimiento de la oferta turística de un destino. En este sentido, la coordinación de éstas a nivel local, regional o nacional es importante para garantizar que los turistas tengan acceso a la información que puedan necesitar tanto del destino España a nivel nacional, como de cada una de las Comunidades Autónomas o municipios.

En el caso concreto de la Comunidad Valenciana, la marca "Red Tourist Info" agrupa a todas las oficinas municipales y las propias de Generalitat para que el turista identifique el servicio como un servicio único en todo el territorio, con el objetivo principal de ofrecer un conjunto integral de servicios. Así, la Red Tourist Info se erige en un instrumento de desarrollo de la política turística orientado a proporcionar un conjunto de servicios de información turística durante todo el año, difundiendo el conocimiento de los recursos y productos de la Comunidad Valenciana, facilitando asistencia y orientación turística, fomentando la hospitalidad y ayudando a optimizar la gestión de la experiencia turística (Ley 15/2018, art.42). No obstante, y a pesar de su importancia, no todas las oficinas de turismo de la Comunidad 
Figura 1: Mapa de la provincia de Alicante con la distribución de oficinas de la Red Tourist Info

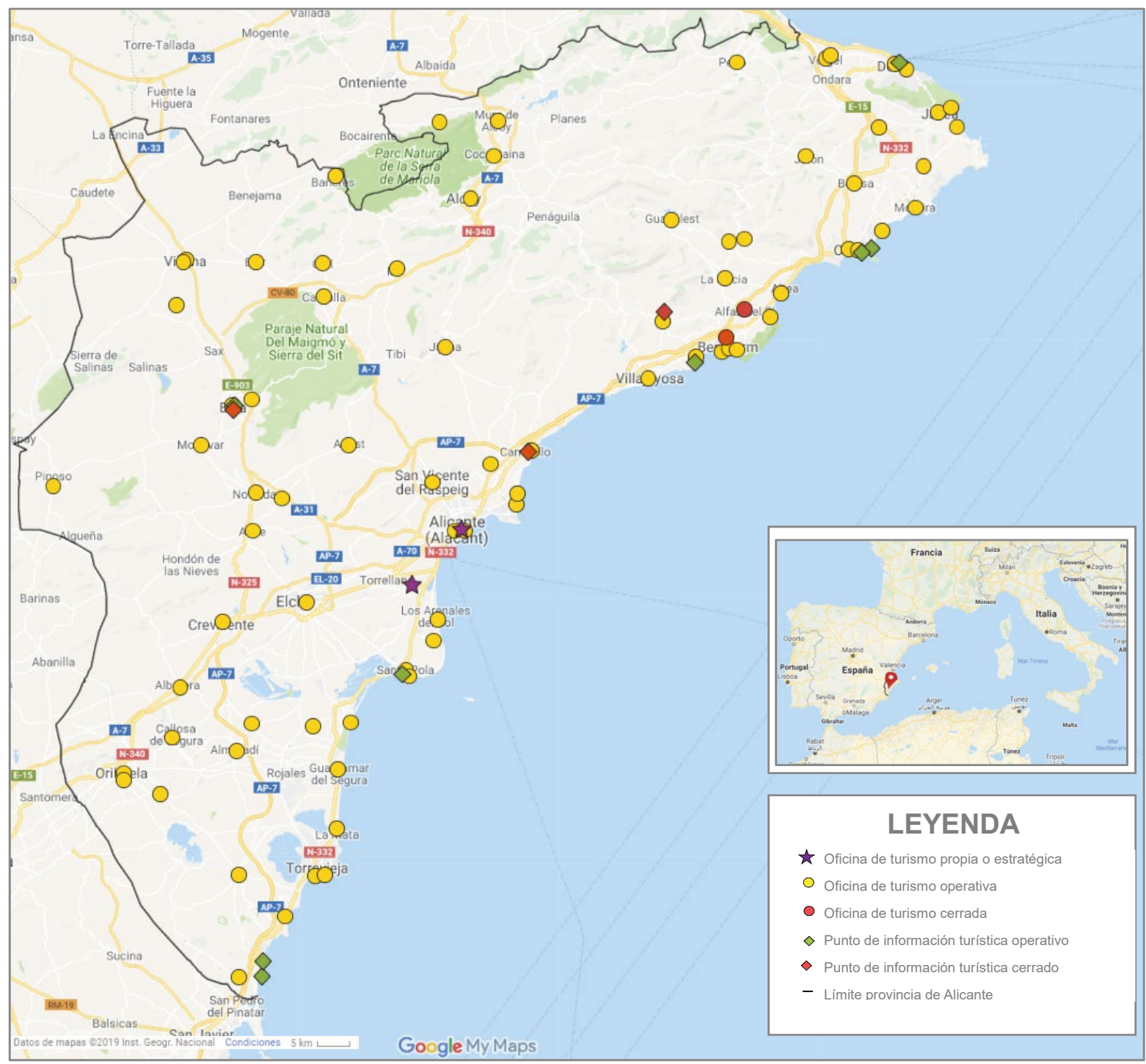

Fuente: elaboración propia con la herramienta "Google My Maps" y los datos de Turisme Comunitat Valenciana (2019).

Valenciana están adheridas a la Red, ya que para poder formar parte de ésta se debe cumplir una serie de requisitos (AVT, 2019) que dificultan el acceso a determinados municipios u oficinas. La "Red" se concibe pues como un instrumento de colaboración con los Ayuntamientos y otras entidades locales, para aunar criterios y rentabilizar esfuerzos y recursos en la gestión de las oficinas de información turística" (Ibañez Jarque, 2001).

Las oficinas municipales son establecimientos que cuentan con unas dependencias estables y dotación básica de mobiliario y equipamiento, y que pueden abrir de manera continuada o con carácter temporal. Por el contrario, los puntos de información suelen ser de carácter temporal y su finalidad es cubrir las necesidades de información turística de los 
municipios adheridos a la Red que, contando con oficinas estables, necesitan acercar la información turística a los principales puntos de interés del destino (eventos, recursos naturales o patrimoniales) donde se concentra un mayor número de turistas durante una determinada época del año.

Específicamente, la provincia de Alicante cuenta con el mayor número de oficinas $(37,91 \%$ del total) y puntos de información $(55,00 \%$ del total) de la Red Tourist Info de la Generalitat Valenciana, alcanzando el 39,24\% del total de los establecimientos de la Comunidad Valenciana adheridos a esta red. En la Figura 1 se presenta un mapa con la distribución geográfica de las diferentes oficinas en la provincia de Alicante.

\subsection{Muestra y metodología}

Con la finalidad de alcanzar los objetivos del trabajo se ha diseñado una investigación de naturaleza cuantitativa a partir de un cuestionario online diseñado específicamente con la plataforma Qualtrics. El cuestionario consta de un total de 28 preguntas cerradas y semiabiertas en el que se obtiene información relativa a los servicios de información turística prestados por las oficinas y puntos de información turística de los diferentes municipios de la provincia de Alicante y adheridos a la Red Tourist Info de la Generalitat Valenciana.

En este sentido, la población objeto de estudio está constituida por la totalidad de oficinas y puntos de información de turismo adheridas a la Red Tourist Info de Generalitat Valenciana en la provincia de Alicante. Dado que determinados municipios de la provincia cuentan con una sola oficina mientras que otros cuentan con varias oficinas o puntos de información adheridos, el análisis se ha realizado considerando esa doble perspectiva: por un lado, a nivel de los municipios de la provincia y, por otro lado, a nivel de oficinas y puntos de información.

La unidad informante a la que va dirigida el cuestionario son los técnicos en turismo $u$ otros responsables de turismo (por ejemplo, concejales $u$ otros cargos del ayuntamiento) que tengan poder de decisión sobre la gestión de las oficinas de turismo del universo objeto del presente estudio. En este punto, conviene recordar la heterogeneidad existente con relación a la gestión de la actividad turística en los diferentes municipios. Mientras que en algunos municipios esta competencia recae en la alcaldía (por ejemplo, Denia) en otros existe una concejalía específica de turismo (por ejemplo, El Campello) o una concejalía con diferentes competencias entre las que está turismo (por ejemplo, Biar). Además, existen municipios con una única oficina o punto de información (por ejemplo, Aspe), mientras que otros cuentan con varias oficinas o puntos (por ejemplo, Torrevieja). De este modo, en primera instancia, se consiguió la colaboración de la coordinación de la Red Tourist Info en la provincia de Alicante, quien remitió un comunicado interno a toda la red informando del estudio en cuestión y quien facilitó para el presente estudio, los contactos directos de los técnicos y/o gestores de las oficinas adheridas a la red. En segunda instancia, se remitió el cuestionario a los contactos directos proporcionados por la Agencia Valenciana de Turismo, indicando que el cuestionario iba dirigido a los técnicos o gestores del servicio de información turística de cada uno de los municipios. Cuando no se obtuvo respuesta, se amplió el plazo de respuesta y se volvió a contactar con los contactos facilitados. De los 50 cuestionarios devueltos, 38 fueron respondidos por técnicos de turismo y 12 por otro tipo de cargos o responsables de turismo. 
Tabla 2: Ficha técnica de la investigación

\begin{tabular}{|r|l|}
\hline Metodología: & $\begin{array}{l}\text { Cuantitativa. Recogida de datos estadísticos en fuentes secundarias + cuestionari- } \\
\text { os online. }\end{array}$ \\
\hline Universo: & 56 municipios y 86 oficinas de turismo adheridas a la Red Tourist Info \\
\hline Ámbito: & Provincia de Alicante. \\
\hline $\begin{array}{r}\text { Tamaño y } \\
\text { diseño muestral: }\end{array}$ & $\begin{array}{l}50 \text { cuestionarios obtenidos para } 50 \text { municipios y } 79 \text { oficinas y puntos de infor- } \\
\text { mación. }\end{array}$ \\
\hline Error muestral* & $\begin{array}{l}\text { Municipios: } \pm 4,6 \%, \text { para un nivel de confianza del } 95 \% . \\
\text { Oficinas y puntos: } \pm 3,2 \% \text { para un nivel de confianza del } 95 \% .\end{array}$ \\
\hline Fechas de campo: & $\begin{array}{l}\text { Obtención de datos cuantitativos de fuentes secundarias. Recogida de datos entre } \\
\text { el } 1 \text { de julio y el } 31 \text { de agosto de } 2019 . \\
\text { Recogida de información de cuestionarios: entre el } 8 \text { de enero y el } 16 \text { de febrero } \\
\text { de } 2020 .\end{array}$ \\
\hline
\end{tabular}

* Asumiendo un muestreo probabilístico y para el peor de los escenarios $(p=q=0,5)$ Elaboración propia.

Específicamente, se ha recogido información sobre las siguientes variables. La información relativa a la externalización se mide a través de una variable dicotómica nominal que toma el valor 1 cuando la gestión de la oficina o punto de información está externalizada en una empresa externa y 0 cuando la gestión es municipal. Esta variable se aproxima a través de la presencia de personal externo en las oficinas o puntos de información. Además, la información sobre la opinión de los responsables se ha medido a través de una serie de variables de intervalo tipo Likert, en las que el encuestado muestra su grado de acuerdo o desacuerdo sobre una serie de afirmaciones en una escala que va desde 1 (totalmente en desacuerdo) a 7 (totalmente de acuerdo). También se ha obtenido información sobre los principales motivos para la externalización del servicio de información turística a través de una variable ordinal.

Además, la información necesaria para alcanzar los objetivos del trabajo se ha complementado con dos fuentes de naturaleza secundaria. En concreto, la información relativa al partido político que gobierna, al partido político que gestiona las competencias en turismo y al tipo de gobernanza de la localidad (mayoría absoluta o coalición) se ha extraído de las bases de datos de la Diputación de Alicante (http://documentacion.diputacionalicante.es/buscacorporacion.asp). La información relativa al partido político que gobierna y el que gestiona las competencias en materia de turismo se ha medido con una variable nominal, mientras que el tipo de gobernanza se mide con una variable nominal dicotómica que toma el valor 1 cuando se gobierna en mayoría y el valor 0 si se gobierna en coalición. Además, se ha utilizado la base de datos WINSITUR que contiene información sobre las características de las diferentes oficinas de turismo.

La recogida de datos primarios se llevó a cabo entre el 8 de enero y el 16 de febrero de 2020. A nivel municipal se ha obtenido una tasa de respuesta del $89,28 \%$, mientras que a nivel del conjunto de las oficinas se ha obtenido una tasa de respuesta del $91,86 \%$. Para el análisis de los datos obtenidos, se han empleado principalmente tres técnicas o métodos de comparación de variables: la distribución T de Student, la prueba U de Mann-Whitney y el test de Ji-Cuadrado. El análisis estadístico se ha realizado con el programa IBM-SPSS v26. 


\section{RESULTADOS}

\subsection{Análisis descriptivo de la situación de las oficinas de turismo en la provincia de Alicante}

En primer lugar, se presenta un breve análisis descriptivo de la situación de las oficinas y puntos de información turísticas adheridas a la Red Tourist Info de Generalitat Valenciana en la provincia de Alicante.

Con relación a la externalización del servicio (medido a través de la presencia de personal externo prestando sus servicios en la red de oficinas de turismo y puntos de información encuestados), los datos indican que en 13 de los 50 municipios incluidos en la muestra existe personal externo ${ }^{2}$, lo que supone el $26 \%$ del total de municipios, mientras que en 37 de los 50 (74\%) no existe personal externo (ver Tabla 3). A nivel de oficinas, los datos señalan que en 17 de las 79 oficinas incluidas en la muestra existe personal externo prestando el servicio, lo que representa el $21,5 \%$ del total de oficinas y puntos de información, mientras que en 62 de 79 (78,5\%) no existe personal externo. Además, y considerando exclusivamente las 17 oficinas externalizadas, se observa que en 9 oficinas (52,9\% del total de oficinas externalizadas) la prestación del servicio por personal externo abarca todo el horario de apertura, en 5 oficinas ( $29,4 \%$ del total) la externalización se produce en fines de semana y festivos, y en 2 oficinas ( $11,8 \%$ del total) la externalización ocurre durante los meses de verano. De una oficina no se ha podido obtener esta información.

Tabla 3: Personal externo en la red de oficinas de turismo a nivel de oficinas y a nivel de municipios.

\begin{tabular}{|c|c|c|c|}
\hline \multicolumn{2}{|c|}{ Nivel municipal } & \multicolumn{2}{c|}{ Nivel oficinas } \\
\hline $\begin{array}{c}\text { Número total de } \\
\text { municipios }\end{array}$ & 56 & $\begin{array}{c}\text { Número total de } \\
\text { oficinas }\end{array}$ & 86 \\
\hline $\begin{array}{c}\text { Número total de } \\
\text { municipios incluidos en } \\
\text { la muestra }\end{array}$ & 50 & $\begin{array}{c}\text { Número total de } \\
\text { oficinas incluidas en la } \\
\text { muestra }\end{array}$ & 79 \\
\hline $\begin{array}{c}\text { Número de municipios } \\
\text { externalizados (\% } \\
\text { sobre el total de la } \\
\text { muestra) }\end{array}$ & $13(26 \%)$ & $\begin{array}{c}\text { Número de oficinas } \\
\text { externalizadas (\% sobre } \\
\text { el total de la muestra) }\end{array}$ & $\begin{array}{c}\text { (9: todo el horario; } \\
5: \text { fines de semana y } \\
\text { festivos; } 2: \text { solo verano; } \\
1: \text { no disponible). }\end{array}$ \\
\hline $\begin{array}{c}\text { Número de municipios } \\
\text { no externalizados (\% } \\
\text { sobre el total de la } \\
\text { muestra) }\end{array}$ & $37(74 \%)$ & $\begin{array}{c}\text { Número de oficinas no } \\
\text { externalizadas (\% sobre } \\
\text { el total de la muestra) }\end{array}$ & 62 (78,5\%) \\
\hline
\end{tabular}

Elaboración propia.

\subsection{Opinión de los encuestados sobre diferentes aspectos sobre la externalización del servicio y la gestión interna por parte de los ayuntamientos}

Dentro del cuestionario, se trató de conocer la opinión de los encuestados con relación a una serie de afirmaciones relativas a diferentes aspectos relativos a la externalización del 
servicio y la gestión interna por parte de los propios ayuntamientos. Esta información proviene indistintamente de los técnicos municipales $u$ otros responsables de turismo en función de la unidad informante, no existiendo diferencias significativas entre ambos tipos de respuesta. Tal y como se ha comentado anteriormente en el apartado 3.2, esta información se obtiene a partir de una serie de variables en las que los encuestados indicaban su grado de acuerdo o desacuerdo sobre una serie de afirmaciones en una escala de 1 a 7. Los resultados parecen confirmar que, en general, los encuestados están ligeramente de acuerdo en afirmar que la externalización del servicio depende de factores políticos (media $=5,29$ ) (ver afirmación 9 de la Tabla 5). Sin embargo, este resultado parece contrastar con el hecho de que cuando se les pregunta a los encuestados sobre los que consideran cuáles son los principales factores de externalización, solo el $3,8 \%$ y el $11 \%$ de los encuestados (en el caso de municipios externalizados y no externalizados, respectivamente) indican que el factor político es uno de los motivos para la externalización del servicio.

Además, los encuestados parecen estar ligeramente de acuerdo en que la contratación de personal municipal para la prestación del servicio es más lenta y complicada administrativamente (media $=5,18$ ) y en que la subcontratación del servicio es mucho más rápido y ágil que la contratación del personal municipal (media $=5,06$ ). En menor medida, el conjunto de encuestados parece indicar que la externalización de personal supone un empeoramiento de las condiciones laborales de los informadores turísticos (media $=4,90$ )

Las afirmaciones referentes a que el servicio prestado por empresas externas es más flexible que si el servicio es prestado por personal municipal, así como que prestar el servicio de información turística con personal municipal es mucho más cómodo que si el servicio se externaliza a una empresa privada y que las oficinas de turismo externalizadas tienen un horario mayor y flexible han obtenido una posición más cercana de la neutralidad que a la confirmación de las mismas, aunque se acercan más a estar ligeramente de acuerdo a estar ligeramente en desacuerdo (medias $=4,14 ; 4,55$ y 4,23 respectivamente).

Además, cabe destacar que la opinión del conjunto de municipios parece no estar del todo de acuerdo con las afirmaciones de que la externalización del servicio a empresas privadas suponga una mejora de la calidad del servicio ofrecido (media $=3,04$ ), así como tampoco con que las oficinas de turismo con personal funcionario no adapten su horario al requerido por los turistas que visitan el municipio (media $=3,71$ ). 
Tabla 5: Consideraciones sobre la prestación del servicio

\begin{tabular}{|l|c|}
\hline \multicolumn{1}{|c|}{ Afirmaciones } & Media \\
\hline $\begin{array}{l}\text { 1-El servicio prestado por empresas externas es más flexible que si el servicio es prestado } \\
\text { por personal municipal }\end{array}$ & 4,14 \\
\hline $\begin{array}{l}\text { 2-Prestar el servicio de información turística con personal municipal es mucho más } \\
\text { cómodo que si el servicio se externaliza a una empresa privada }\end{array}$ & 4,55 \\
\hline $\begin{array}{l}\text { 3- La contratación de personal municipal para la prestación del servicio es más lento y } \\
\text { complicado administrativamente. }\end{array}$ & 5,18 \\
\hline 4- Las oficinas de turismo externalizadas tienen un horario mayor y flexible. & 4,23 \\
\hline $\begin{array}{l}\text { 5-Las oficinas de turismo con personal funcionario no adaptan su horario al requerido por } \\
\text { los turistas que visitan el municipio (fines de semana y festivos). }\end{array}$ & 3,71 \\
\hline $\begin{array}{l}\text { 6-La externalización del servicio a empresas privadas supone una mejora de la calidad del } \\
\text { servicio ofrecido. }\end{array}$ & 3,04 \\
\hline $\begin{array}{l}\text { 7-La externalización de personal supone un empeoramiento de las condiciones laborales } \\
\text { de los informadores turísticos. }\end{array}$ & 4,90 \\
\hline $\begin{array}{l}\text { 8-La subcontratación del servicio es mucho más rápida y ágil que la contratación de } \\
\text { personal municipal. }\end{array}$ & 5,06 \\
\hline 9-La externalización del servicio depende de factores políticos. & 5,29 \\
\hline
\end{tabular}

Elaboración propia.

En cuanto a los principales motivos para la externalización del servicio de información turística, los encuestados indican que la necesidad de un horario mayor y flexible $(34,6 \%)$ es el principal motivo, seguido de la falta de un plan de empleo público en el Ayuntamiento que permita disponer de personal funcionario $(30,80 \%)$ y de la mayor facilidad burocrática en tercer lugar (11,5\%). Según los encuestados, a estos motivos le seguirían, en menor medida, la reducción de costes del servicio $(7,7 \%)$ y, por último, con un $3,8 \%$ y en la misma proporción, los factores políticos, la inexistencia de técnico en turismo en el municipio, la mayor especialización del personal empleado y otros factores.

\subsection{Propensión a externalizar en función de factores políticos}

En este apartado se analiza la propensión a externalizar el servicio de información turística en función del partido político que sustenta la alcaldía (partido que gobierna). Así, la tabla 6 muestra los partidos políticos que ostentan las diferentes alcaldías de los 50 municipios objeto del presente estudio, indicando la proporción de Ayuntamientos liderados en alcaldía por un determinado partido político que externalizan el servicio y los municipios liderados por ese mismo partido político que no externalizan el servicio. 
Tabla 6: Propensión a externalizar según partido político en alcaldía

\begin{tabular}{|ccccc|}
\hline \multirow{2}{*}{ ALCALDía } & & \multicolumn{2}{c}{ ¿Existe personal externo? } & \\
\cline { 3 - 4 } & & NO & SI & Total \\
\hline EUPV & Observado & 2 & 0 & 2 \\
& \% dentro de la fila & $100,0 \%$ & $0,0 \%$ & $100,0 \%$ \\
COMPROMís & Observado & 6 & 1 & 7 \\
& \% dentro de la fila & $85,7 \%$ & $14,3 \%$ & $100,0 \%$ \\
PSOE & Observado & 15 & 6 & 21 \\
CIUDADANOS & \% dentro de la fila & $71,4 \%$ & $28,6 \%$ & $100,0 \%$ \\
& Observado & 2 & 0 & 2 \\
PPP & \% dentro de la fila & $100,0 \%$ & $0,0 \%$ & $100,0 \%$ \\
& Observado & 11 & 6 & 17 \\
OTROS & \% dentro de la fila & $64,7 \%$ & $35,3 \%$ & $100,0 \%$ \\
PARTIDOS & Observado & 1 & 0 & 1 \\
TOTAL & \% dentro de la fila & $100,0 \%$ & $0,0 \%$ & $100,0 \%$ \\
& Observado & 37 & 13 & 50 \\
& $\%$ dentro de la fila & $74,0 \%$ & $26,0 \%$ & $100,0 \%$ \\
\hline
\end{tabular}

Elaboración propia.

Del total de los municipios, el 26\% (13 municipios) han externalizado el servicio. Para contrastar las diferencias entre esa externalización entre partidos, y al comparar el porcentaje de externalización por partido, vemos que los dos grandes partidos que sustentan las alcaldías (PP y PSOE) y que externalizan el servicio gobiernan en total 12 de los 13 municipios externalizados. En ambos casos, obtienen unos porcentajes similares del 28,6\% (6 municipios de 21) en el caso del PSOE y del 35,3\% (6 municipios de 17) en el caso del PP. Además, esas diferencias no son estadísticamente significativas según la prueba de la ji-cuadrado (valor=3,09, con un p-valor de 0,686 $(>0,05))$, por lo que se puede aceptar que la propensión a externalizar es similar entre ambos partidos.

Con relación al partido político que gestiona la concejalía de turismo, y al igual que en el caso anterior, los resultados indican que no existen diferencias significativas entre la propensión a externalizar según dicho partido. En este caso, la prueba de Ji-cuadrado ofrece un valor de 2,35 ( $p$-valor de $0,799(>0,05))$, que indica que no existen diferencias significativas o relevantes como para confirmar que este tipo de factores políticos influyan en la propensión a externalizar. 
Tabla 7: Propensión a externalizar según partido político en concejalía turismo

\begin{tabular}{|lccc|}
\hline & \multicolumn{2}{c|}{ ¿Existe personal externo? } & \\
\cline { 2 - 3 } PARTIDO CONCEJALÍA TURISMO & NO & SI & Total \\
\hline EUPV & & & \\
COMPROMÍS & 2 & 0 & 2 \\
PSOE & 3 & 2 & 5 \\
CIUDADANOS & 17 & 5 & 22 \\
PP & 2 & 1 & 3 \\
OTROS PARTIDOS & 11 & 5 & 16 \\
Total & 2 & 0 & 2 \\
\hline
\end{tabular}

Elaboración propia.

Sin embargo, si analizamos la propensión a externalizar en función del tipo de gobernanza, esto es, si se tiene mayoría absoluta o se gobierna en coalición o alianza con otros partidos, los datos son relevantes y parecen indicarnos que los gobiernos municipales que cuentan con mayoría absoluta tienden más a la externalización que aquellos que están en coalición o minoría y dependen de pactos de gobierno. En este caso, el factor político viene determinado ya no por el color de la alcaldía o el partido que sustenta las competencias turísticas, sino por la composición del órgano de gobierno, tendiendo más a externalizar aquellos municipios que gobiernan en mayoría absoluta que aquellos que están gobernando con pactos o alianzas. Así, de los 29 municipios en lo que se gobierna en mayoría absoluta, 11 (el 37,9\%) tienden a la externalización del servicio, mientras que de los 21 en los que se gobierna en alianza solo $2(9,5 \%)$ externalizan el servicio de información turística. De este modo, se observa que de los 13 municipios que externalizan el servicio de información, en 11 se gobierna en mayoría absoluta mientras que solo en 2 se gobierna coalición o alianza. Comparando las proporciones en ambos casos, podemos observar que la igualdad de proporciones no se cumple en este caso, existiendo una mayor propensión a externalizar en los municipios gobernados en mayoría absoluta (11 de 29 municipios) y menos propensión en los gobernados mediante pactos o alianzas (2 de 21 municipios). La prueba de Ji-cuadrado sobre la propensión a externalizar en función del tipo de gobernanza parece corroborar significativamente estos resultados (valor del estadístico de 5,11, con un p-valor de 0,024 inferior a 0,05), lo que permite rechazar la igualdad de proporciones y afirmar que los Ayuntamientos donde hay mayoría absoluta el servicio de información turística se externaliza en mayor proporción que donde hay alianzas o coaliciones. 
Tabla 8: Propensión a externalizar de municipios con mayoría absoluta frente a municipios con gobiernos de coalición

\begin{tabular}{|c|c|c|c|c|}
\hline & & \multicolumn{2}{|c|}{ ¿Existe personal externo? } & \multirow[b]{2}{*}{ Total } \\
\hline \multicolumn{2}{|c|}{ ¿Mayoría absoluta o alianza? } & No & Si & \\
\hline \multirow[t]{2}{*}{$\begin{array}{l}\text { Mayoría } \\
\text { Absoluta }\end{array}$} & Observado & 18 & 11 & 29 \\
\hline & $\%$ dentro de la fila & $62,1 \%$ & $37,9 \%$ & $100,0 \%$ \\
\hline \multirow[t]{2}{*}{ Alianza } & Observado & 19 & 2 & 21 \\
\hline & $\%$ dentro de la fila & $90,5 \%$ & $9,5 \%$ & $100,0 \%$ \\
\hline \multirow[t]{2}{*}{ Total } & Observado & 37 & 13 & 50 \\
\hline & $\%$ dentro de la fila & $74,0 \%$ & $26,0 \%$ & $100,0 \%$ \\
\hline
\end{tabular}

Elaboración propia.

\section{CONCLUSIONES}

La externalización de servicios de información turística es una realidad en la provincia de Alicante. Así lo demuestran los datos obtenidos, donde el $26 \%$ de los municipios que han participado en el estudio han externalizado total o parcialmente el servicio de información turística municipal, representando en total al $21,5 \%$ de las oficinas y puntos de información de la provincia.

Aunque los resultados parecen indicar que los factores políticos no tienen una gran incidencia en la externalización del servicio de información turística, dado que este hecho no depende ni del partido político que gobierna, ni del partido que sustenta la competencia en turismo, los resultados de este estudio evidencian que este tipo de factores sí son importantes, puesto que el hecho de que se disponga de mayoría absoluta o no sí ejerce una influencia sobre la propensión a externalizar. Además, en el análisis de las opiniones de los encuestados sobre la prestación del servicio, los factores políticos han obtenido una media de 5,29 sobre 7 en la escala de Likert, habiendo sido escogido como el principal factor o motivo por el que se externaliza el servicio de información turística en la provincia de Alicante frente a otras afirmaciones y factores.

De hecho, en cuanto a la influencia de los factores políticos a la hora de externalizar el servicio de información turística, los resultados obtenidos con el presente trabajo de investigación parece indicarnos que no existe una mayor o menor propensión a externalizar en base al partido político que regenta la alcaldía de los municipios alicantinos, ni en base al partido que tiene las competencias en materia de turismo dentro de las corporaciones municipales, no existiendo diferencias significativas en la propensión de cada uno de ellos según los datos obtenidos. Sin embargo, los resultados señalan que la forma de gobernanza si ejerce una influencia, de forma que los gobiernos municipales que disponen de mayoría absoluta (independientemente del color político), tienden a una mayor externalización del servicio de información turística frente a aquellas corporaciones que gobiernan en coalición, con independencia del partido político que gobierne. 
En cuanto a la implicación del estudio realizado cabe destacar dos grandes contribuciones. En primer lugar, el presente trabajo se configura como el primer proyecto de investigación, al menos entre los conocidos por los autores, que se centra en analizar la influencia de los factores políticos en la externalización de los servicios de información turística. En segundo lugar, cabe señalar que conocer los principales motivos de los gestores turísticos para la externalización del servicio puede ayudar a éstos a la hora de tomar la decisión sobre si externalizar o no, ya que la mayoría de los municipios que han licitado el servicio han alegado la necesidad de disponer de un horario mayor y flexible, la falta de un plan de empleo público municipal y la mayor facilidad burocrática.

Entre las limitaciones del trabajo cabe destacar las siguientes. En primer lugar, que el presente estudio se circunscribe únicamente al servicio de información turística adherido a la Red Tourist Info de Generalitat Valenciana en la provincia de Alicante, por lo que el estudio se limita únicamente a esta área geográfica y a las oficinas y municipios adheridos a la Red, dejando fuera oficinas municipales locales que no están adheridas a la Red Tourist Info. En segundo lugar, la externalización se ha medido, exclusivamente, valorando la presencia física de personal externo aportado por las empresas adjudicatarias del servicio a las oficinas o puntos de información turística, sin valorar otras formas de externalización del servicio como podría ser la gestión de la información que se proporciona en canales digitales, asistencia a ferias, etc.

A partir del presente estudio, y como futuras líneas de investigación, se podría ampliar el presente estudio a toda la Comunidad Valenciana para conocer si los factores políticos influyen de la misma manera en las 3 provincias y si la externalización de las oficinas y puntos de información turística de la red se produce por igual en todo el ámbito autonómico, ya que la realidad turística de la provincia de Alicante no es la misma que la de Valencia o Castellón. Podría resultar interesante ampliar el estudio al resto de provincias valencianas y compararlo con los datos que arroje a nivel autonómico, para identificar si se sigue el mismo patrón en las 3 provincias o si los datos arrojados por cada provincia son distintos.

Otra futura línea de investigación, podría centrarse en ampliar el objeto del estudio a otro tipo de servicios turísticos prestados por las administraciones públicas a nivel municipal, autonómico y estatal como por ejemplo, el servicio de promoción turística, que a su vez se divide en servicios específicos como la promoción online (web y redes sociales), la asistencia a ferias y eventos internacionales relacionados con turismo, promociones en destinos emisores de turistas (fam-trips, presentaciones en destino,...), etc, analizando si los factores políticos influyen o no a la hora de externalizar otro tipo de servicios turísticos. 


\section{REFERENCIAS BIBLIOGRÁFICAS}

AVT (2019). Llistat de municipis de la comunitat valenciana declarats turístics. Valencia: Agència Valenciana del Turisme. Recuperado de http://www.turisme.gva.es/turisme/es/files/ pdf/Listado Municipios CV declarados turisticos.pdf

Bel, G. (2009). El dilema entre público y privado en la gestión de los servicios públicos. En C. Ramió (Coordinador), La colaboración público-privada y la creación de valor público (pp.39-56). Barcelona: Diputación de Barcelona

Comunidad Valenciana. Ley 8/2010, de 23 de junio, de régimen local de la Comunitat Valenciana. Boletín Oficial del Estado, núm.178, de 23 de julio de 2010, pp.64224-64288. Recuperado de https://www.boe.es/boe/dias/2010/07/23/pdfs/BOE-A-2010-11729.pdf

Comunidad Valenciana. Ley 15/2018, de 7 de junio, de turismo, ocio y hospitalidad de la Comunidad Valenciana. Boletín Oficial del Estado, núm.157, de 29 de junio de 2018, pp.65200-65258. Recuperado de https://www.boe.es/boe/dias/2018/06/29/pdfs/ BOE-A-2018-8950.pdf

España. Constitución Española de 1978. Boletín Oficial del Estado, núm. 311, de 29 de diciembre de 1978, pp.29315-29424. Actualización de 27 de noviembre de 2011. Recuperado de https://www.boe.es/buscar/pdf/1978/BOE-A-1978-31229-consolidado.pdf

España. Ley 7/1985, de 2 de abril, reguladora de las Bases del Régimen Local. Boletín Oficial del Estado, núm. 80, de 3 de abril de 1985, pp.8945-8964. Texto Consolidado a 4 de agosto de 2018. Recuperado de https://www.boe.es/buscar/pdf/1985/BOE-A-19855392-consolidado.pdf

España. Ley 9/2017, de 8 de noviembre, de Contratos del Sector Público, por la que se transponen al ordenamiento jurídico español las Directivas del Parlamento Europeo y del consejo 2014/23/UE y 2014/24/UE, de 26 de febrero de 2014. Boletín Oficial del Estado, núm.272, de 9 de noviembre de 2017, pp.107714-108007. Recuperado de https:// www.boe.es/boe/dias/2017/11/09/pdfs/BOE-A-2017-12902.pdf

España. Tribunal Constitucional (Pleno). Conflicto positivo de competencias 860-1983, 862 y $865 / 1983$. Sentencia $125 / 1984$, de 20 de diciembre. Boletín Oficial del Estado, núm.10, de 11 de enero de 1985, pp.19-22. Recuperado de https://www.boe.es/boe/ dias/1985/01/11/pdfs/T00019-00022.pdf

Fernández Rodríguez, C. (2016). Derecho administrativo del turismo. Barcelona: Marcial Pons. Garulo, C., Díez, J.J., y Sellers, R. (octubre 2020). Comunicación Influencia de la Covid-19 en la tendencia a la externalización de servicios de información turística. En Rafael Lapiedra (Presidencia), XXIII Congreso Internacional de Turismo Universidad y Empresa: "Turismo: Nuevo paradigma tras la Covid-19". Castellón.

Guerra, R.P., y Martín, M.M.C. (2009). La configuración del Derecho del turismo autonómico español en el ordenamiento jurídico administrativo-constitucional. Revista Aragonesa de Administración Pública, (35), 455-518.

Ibáñez Jarque, C. (2001). Servicios de Información al Turista sobre el Territorio: la Red Tourist Info y el Plan de Señalización Turística de la Comunidad Valenciana. Métodos de información, 8 (42), 60-68. 
INE (2018). Nota de prensa Cuentas Satélite del Turismo de España (CST). Revisión Estadística 2019. Serie 2016-2018. Recuperado de https://www.ine.es/prensa/cst 2018.pdf

Junior Report (7 de noviembre de 2019) ¿Qué define la derecha y la izquierda? La Vanguardia. Recuperado de https://www.lavanguardia.com/vida/ junior-report/20181019/452426477946/dercha-izquierda-politica-ideologia-derechos-sociales-empresa.html

Laguna de Paz, J.C. (2011). Regulación, externalización de actividades administrativas y autorregulación. Revista de Administración Pública, núm.185, 89-112. Recuperado de https://dialnet.unirioja.es/descarga/articulo/3732677.pdf

Martínez Rodríguez, J. (2003). Los determinantes de la contratación externa de servicios públicos locales. En J.R. Cuadrado Roura (Presidencia), XXIX Reunión de Estudios Regionales. Reunión llevada a cabo en la reunión de la Asociación Española de Ciencia Regional, Santander.

Mirabell Izard, O. (2011). Gestión de oficinas de turismo (Vol.82). Barcelona: Editorial UOC.

Morales Caraballo, M.G. (2018). Servicios de Información turística. Madrid: Ediciones Paraninfo, S.A.

OMT-UNWTO. (2018). Panorama OMT del turismo internacional. Edición 2018. Madrid. Organización Mundial del Turismo (OMT-UNWTO). Recuperado de https://www.eunwto.org/doi/book/10.18111/9789284419890

Pina, V., y Torres, L. (2002). Descentralización/Externalización de Servicios y Consolidación de Cuentas en las Corporaciones Locales. Spanish Journal of Finance and Accounting / Revista Española de Financiación y Contabilidad, 31 (111), 225-244.

Ramió, C. y García Codina, O. (2006). La externalización de servicios públicos en España: la necesidad de repensar una nueva gestión pública planificada, controlada y evaluada. Revista del CLAD Reforma y Democracia, (35) 1-22.

Ramió, C. y Salvador, M.(2012). Provisión de servicios públicos en el contexto de la administración local de España. El papel de los factores políticos institucionales y la externalización de los gobiernos locales. Gestión y Política Pública. Vol.XXI (2), 375-405.

Rodríguez-Arana, J. (2008). Sobre la distribución de competencias en materia de turismo. Revista aragonesa de administración pública, (32) 369-406.

Salvador, M. y Riba, C. (2017). Outsourcing as management strategy in local public services. Brazilian Journal of Public Administration. Vol.54 (4), 633-652.

Turisme Comunitat Valenciana (2019). Listado de Oficinas de Turismo de la Red Tourist Info de Generalitat Valenciana. Consulta programa WINSITUR a fecha 17 de julio de 2019.

Unión Europea. Dictamen del Comité de las Regiones sobre "El papel de las autoridades locales y regionales en el desarrollo turístico y la intervención de la Unión Europea en materia de turismo>>. Diario Oficial de la Unión Europea C 293, 13 de octubre de 1999, p.0033. Recuperado de https://eur-lex.europa.eu/legal-content/ES/ TXT/?uri=uriserv:OJ.C . 1999.293.01.0033.01.SPA\&toc=OJ:C:1999:293:TOC

Valencia, A. (2019). Externalización de servicios públicos y Nueva Gestión Pública: la perspectiva de la Ciencia Política. En Vila, F.A (Coordinador), Público y Privado en la gestión de los servicios públicos: reestructuración, externalización y reversión a la Administración (pp. 17-25). Madrid: Instituto Nacional de Administración Pública. 
Villanueva Cuevas (2016). Las competencias de los entes locales en materia de turismo tras la reforma del régimen local español. En C. Tapia, Carrasco y Oliva (coordinadores). Hacia el ámbito del derecho administrativo. (pp.167-182). México: Ediciones Eternos Malabares.

\section{CONTRIBUCIÓN DE LOS AUTORES}

Los tres autores han contribuido de forma equitativa en el desarrollo del trabajo.

\section{AGRADECIMIENTOS}

El presente estudio no ha sido financiado por ninguna agencia. 